\title{
Aux médecins de premier recours
}

Chères et chers collègues,

Nous sommes, à mi-décembre, à 6 mois d'expérience avec le TARMED LAA ... et à deux semaines de l'introduction du TARMED LAMal. Les premières expériences sont faites, le tarif lui-même s'apprivoise petit à petit, et son emploi généralisé pour toutes nos consultations ne devrait finalement pas poser trop de problèmes.

Une nouvelle est cependant tombée il y a deux semaines qui doit absolument nous alerter: le prix moyen par cas de nos traitements «accident» est en train de dépasser de loin les normes fixées pour la neutralité des coûts!

De quoi s'agit-il? Ça n'est pas compliqué...

Il a été imposé depuis toujours par les autorités politiques que l'introduction du TARMED ne soit en aucun cas l'occasion d'une augmentation globale des coûts; en gros, pour l'ensemble du corps médical, le coût moyen par cas pendant les 18 premiers mois du nouveau tarif ne doit ainsi pas être plus élevé qu'il ne l'a été ces dernières années.

Or depuis deux mois, nos factures, à nous médecins de premier recours, dépassent très largement cette limite!

Certes, il était prévu - et souhaitable - que le TARMED permette une revalorisation de nos prestations «intellectuelles» par rapport aux prestations «techniques» des spécialistes, mais là, cela va trop loin! Nous sommes en train de tellement tirer les coûts vers le haut qu'une baisse de la valeur du point risque de devenir inévitable.

Pour éviter une telle mesure, qui pénaliserait l'ensemble du corps médical, ou pour éviter des mesures qui nous pénaliseraient spécifiquement nous, médecins de premier recours, les Présidents de la SSMG, de la SSMI et de la SSP ont rencontré la Commission d'évaluation TARMED le 12 décembre dernier, pour discuter des mesures à prendre.

Nous allons évidemment nous battre tant qu'il faudra pour défendre nos positions.

Mais vous êtes toutes et tous impliqué-es aussi: nous avons trois mois, pas davantage, pour faire la preuve que le point à Fr 1.- peut être maintenu!

Il semble en fait y avoir des explications techniques assez simples au dépassement constaté - c'est ce que Jürg Pellaton explique dans les pages qui suivent et que nous vous invitons à lire avec la plus grande attention.

Il semble ainsi parfaitement possible de garder la valeur du point accident à Fr 1.-, et donc de préserver les avantages que nous, médecins de premier recours, savons trouver dans le TARMED - mais il faut pour cela que chacune et chacun d'entre nous, généralistes, internistes et pédiatres, s'en tienne à une utilisation adéquate du tarif.

Nous vous remercions de tout ce que vous ferez dans ce sens, dans les mois qui viennent, faisant ainsi la preuve de notre crédibilité à toutes et à tous, et de notre maturité.

Jacques de Haller, Président de la SSMG

Werner Bauer, Président de la SSMI

Pierre Klauser, Président de la SGP

Marc Müller, Président du CMPR 